\title{
Quantum control of nano-particles at catalysis surface
}

Quan-Fang Wang*

Chinese University of Hong Kong, Shatin, N.T., Hong Kong

\begin{abstract}
Quantum control is always interested in all the phenomena in the word. ${ }^{1}$ At the nano-scale, particle at catalysis surface is a research topic in connected of quantum mechanics and surface science. Nano-particles appeared at a certain crystals would be considered as control object in this regarding. Theoretical issue is taking account into control using quantum control theory. An exciting conclusion is attractive in this work ultimately.
\end{abstract}

\section{History of nano particle}

Nano particle indicate a particle in the scale of $10^{-9} \mathrm{~m}$, it is intermediate particle of atom and molecule. ${ }^{2}$ Those particles possess unique properties of electronic, magnetic, mechanics, conduction, physical, optical than other particles. For unit nanometer $1 \mathrm{~nm}=10^{-9} \mathrm{~m}$, a particle usual at $1 \mathrm{~nm}$ to $100 \mathrm{~nm}$. Early nanoparticle appeared in ancient materials, surface of ceramic. After it had been recognized as nanoparticle, people had discovered carbon (C) nanotube, nanowire, nanomotor, nanorod as advance materials. Significantly, nanotechnology had being utilized in industry, aerospace, medicine and other areas.

\footnotetext{
${ }^{*}$ Corresponding author: Dr. Quan-Fang Wang quanfangwang9@gmail.com
} 
As nanopartice on a matter, metalic or catalysis surface, researches had been reported on the reaction and non-reaction cases. First is nanoparticle take chemical interaction at surface; second is the nanoparticle non-reacted on surface in a magnetic field. At a certain nanocrystal surface (such as silicon crystal for memory storage), nanoparticle can be growth in lab experiments. Control of nanoparticle had been considered for physical purpose to make an ideal nano-material. In general, at current instrument equipments (TEM, STM), those kinds of control can be occurred for adjustment of temperature, pressure, particle-type, and so forth. Theoretically, control of nanoparticle at a practical crystal surface is a fairly interesting topic: what dynamics are those particle obey, what can we do for a nanoparticle, can one control the nanoparticle as they are on the surface.

In this work, nanoparticle at surface is considered as control target. Control theory is applied to a quantum system (named as nanosystem) of nanoparticle in the form of time-depended Schrödinger equation of electron density function. Apart from Schrödinger equation of wave function for quantum physics, density functional theory is proposed in early years of last century. ${ }^{3}$ Different to represent a elementary particle as wave function, the idea of density functional theory is laying on representing a quantum particle by electron density. In this theoretic work, density functional theory (DFT) is using as tool to do control at the framework of Thomas-Fermi (TF) theory. ${ }^{4,5}$ TF model had already been modified as Thomas-Fermi-Dirac (TFD) model, ${ }^{6}$ ThomasFermi-von Weizsäcker (TFW) model, ${ }^{7}$ Hohenberg-Kohn model for one electron, ${ }^{8}$ Kohn-Sham model for including exchange and correlation correction. ${ }^{9}$

\section{Density function theory}

For nanoparticle, roughly introduce density functional theory as preparation. ${ }^{10}$ Let $\Omega$ be an open bounded set of $\mathbf{R}^{3}$ at spherical polar coordinate $\mathbf{r} \in(0, \infty)$, and $Q=(0, T) \times \Omega$ for $T>0,(t, \mathbf{r}) \in Q$. The ground state energy density of electron is denoted as $n(t, \mathbf{r}), \mathbf{r}$ is the displacement of electron from centre of nanoparticle, $\vec{E}(t, \overrightarrow{\mathbf{r}})$ is electronic field. Then time-dependent density functional 
theory is taking the form of Schödinger equation as ${ }^{11}$

$$
\mathbf{i} \frac{\partial}{\partial t} \eta(t, \mathbf{r})=\left[-\frac{1}{2} \nabla^{2}+\int \frac{n\left(t, \mathbf{r}^{\prime}\right)}{\left|\mathbf{r}-\mathbf{r}^{\prime}\right|} d \mathbf{r}^{\prime}+\frac{\delta E_{x c}(n(t, \mathbf{r}))}{\delta n(t, \mathbf{r})}-\vec{E}_{0}(t) \cdot \overrightarrow{\mathbf{r}}\right] \eta(t, \mathbf{r})
$$

In here, $\eta(t, \mathbf{r})$ is wave function of the particle, represent the probability function at time $t$. $E_{x c}(n(t, \mathbf{r}))$ is exchange correction function. For unified scattered field $\vec{E}_{S}(t, \overrightarrow{\mathbf{r}})$, the Hamiltonian of nanoparticle in the presence of incident field $\vec{E}_{0}(t)$ at time-depended density functional theory (TDDFT) lead the form of

$$
\hat{H}(t)=-\frac{1}{2} \nabla^{2}+\int \frac{n\left(t, \mathbf{r}^{\prime}\right)}{\left|\mathbf{r}-\mathbf{r}^{\prime}\right|} d \mathbf{r}^{\prime}+\frac{\delta E_{x c}(n(t, \mathbf{r}))}{\delta n(t, \mathbf{r})}-\vec{E}_{0}(t) \cdot \overrightarrow{\mathbf{r}}-\vec{E}_{s}(t, \mathbf{r}) \cdot \overrightarrow{\mathbf{r}}
$$

\section{Time depended Schrödinger equation}

Practically, for $N$ nanoparticle at surface, $n\left(t, \mathbf{r}^{\prime}\right)=\sum_{i=1}^{N}\left|\eta^{i}\left(t, \mathbf{r}^{\prime}\right)\right|^{2}$, time-depended Schrödinger equation is expressed as

$$
\left\{\begin{array}{l}
\mathbf{i} \frac{\partial}{\partial t} \eta^{i}(t, \mathbf{r})=-\frac{1}{2} \Delta \eta^{i}(t, \mathbf{r})+[e(t, \mathbf{r})+V(t, \mathbf{r})-g(t)] \eta^{i}(t, \mathbf{r}) \\
\eta^{i}(0, \mathbf{r})=\eta_{0}^{i}(\mathbf{r}), \quad 1 \leq i \leq N
\end{array}\right.
$$

System (1) is said as nanosystem. In here, $e(t, \mathbf{r})$ is electron-electron interaction, $V(t, \mathbf{r})$ is contribution of potential energy functional $E_{x c}$, and $g(t)$ is external electronic field.

$$
e(t, \mathbf{r})=\int \frac{n\left(t, \mathbf{r}^{\prime}\right)}{\left|\mathbf{r}-\mathbf{r}^{\prime}\right|} d \mathbf{r}^{\prime}, V(t, \mathbf{r})=\frac{\delta E_{x c}(n(t, \mathbf{r})}{\delta n(t, \mathbf{r})}, g(t)=\vec{E}_{0}(t) \cdot \overrightarrow{\mathbf{r}}
$$

For different density functional model, $E_{x c}$ at $V(t, \mathbf{r})$ is expressed as detail

$$
\begin{aligned}
V(t, \mathbf{r}) & =\frac{\delta E_{x c}(n(t, \mathbf{r}))}{\delta n(t, \mathbf{r})} \\
& =\frac{5}{3} c_{k} \int n(\mathbf{r})^{2 / 3} d \mathbf{r}+v(\mathbf{r}) \quad \text { TF model }
\end{aligned}
$$




$$
\begin{array}{rlr}
= & \frac{5}{3} c_{k} \int n(\mathbf{r})^{2 / 3} d \mathbf{r}+v(\mathbf{r})-\frac{4}{3} c_{e} \int n(\mathbf{r})^{\frac{1}{3}} d \mathbf{r} & \text { TFD model } \\
= & \frac{5}{3} c_{k} \int n(\mathbf{r})^{2 / 3} d \mathbf{r}+v(\mathbf{r})-\frac{4}{3} c_{e} \int n(\mathbf{r})^{\frac{1}{3}} d \mathbf{r} & \\
& +\frac{1}{8}\left(\frac{h^{2}}{m}\right) \int\left[\frac{|\tilde{N} n(\mathbf{r})|^{2}}{n^{2}(\mathbf{r})}-2 \frac{\tilde{N}^{2} n(\mathbf{r})}{n(\mathbf{r})}\right] d \mathbf{r}, & \text { TFDW model }
\end{array}
$$

$c_{k}=\frac{3}{10}\left(\frac{h}{m}\right)\left(3 \pi^{2}\right)^{2 / 3}$ and $c_{e}=e^{2} \frac{3}{4}\left(\frac{3}{\pi}\right)^{1 / 3}$. For several nucleus, coulomb potential $v(\mathbf{r})=\sum_{\sigma} \frac{C_{\sigma}}{\left|\mathbf{r}^{i}-R_{\sigma}\right|}$, $C_{\sigma}$ is charge, $\mathbf{r}^{i}$ is coordinate of electron at nucleus $R_{\sigma}$. At TFW model, last term of TFDW theory is in the form of gradient expression as $\frac{\hbar}{m} \int\left|\left(\nabla n^{1 / 2}\right)(\mathbf{r})\right|^{2} d \mathbf{r}$. The equation in nano system (1) can be called as nano equation.

\section{Mathematical setting}

To do mathematical setting for time-depended Schrödinger equation (1) in the framework of variational method. For the practice of nanoparticle at matter or metallic surface, neglect the consideration of complex space for wave function $\eta^{i}(t, \mathbf{r})$ on variable $t$ and $\mathbf{r}$, take real Hilbert space for real part and imaginary part in variational theory. In nano system $(1), g(t)$ is external force of electron, external control input of nanoparticle $P^{i}$. Control $g(t)$ is only depended on time $t$, and independent of spatial variable $\mathbf{r}$.

For real part and imaginary part of complex-valued function $\eta^{i}(t, \mathbf{r})$, consider two Hilbert spaces $H=L^{2}(\Omega)$ and $V=H_{0}^{1}(\Omega)$ for usual norm and inner product. ${ }^{12} V^{\prime}$ denotes conjugate space of $V$. Actually, $(V, H)$ is a Gelfand triple spaces $V \hookrightarrow H \hookrightarrow V^{\prime}$, two embeddings are continuous, dense and compact. For $\eta^{i}=\eta^{i 1}+\mathbf{i} \eta^{i 2}, \eta^{i 1}, \eta^{i 2} \in L^{2}(\Omega)$, norm of $\eta^{i 1}, \eta^{i 2}$ in space $L^{2}(\Omega), H_{0}^{1}(\Omega)$ as $\|\cdot\|_{H},\|\cdot\|_{V}$. For $\eta^{i}=\eta^{i 1}+\mathbf{i} \eta^{i 2}$, then inner product of $\eta^{i 1}$ and $\eta^{i 2}$ in space $L^{2}(\Omega)$ and $H_{0}^{1}(\Omega)$ denoted as $(\cdot, \cdot)_{H}$ and $(\cdot, \cdot)_{V}$. In here, $\eta^{i^{\prime}}=\frac{\partial \eta^{i}}{\partial t}$.

Definition 1. For $\eta^{i}=\eta^{i 1}+\mathbf{i} \eta^{i 2}$, define solution space $W(0, T)$ as

$$
W(0, T)=\left\{\eta^{i} \mid \eta^{i 1}, \eta^{i 2} \in L^{2}(0, T ; V), \eta^{i 1^{\prime}}, \eta^{i 2^{\prime}} \in L^{2}\left(0, T ; V^{\prime}\right)\right\}
$$


The norm of $\eta^{i}$ in the space $W(0, T)$ can be defined as

$$
\left\|\eta^{i}\right\|_{W(0, T)}=\left(\left\|\eta^{i 1}\right\|_{L^{2}(0, T ; V)}^{2}+\left\|\eta^{i 2}\right\|_{L^{2}(0, T ; V)}^{2}+\left\|\eta^{i 1^{\prime}}\right\|_{L^{2}\left(0, T ; V^{\prime}\right)}^{2}+\left\|\eta^{i 2^{\prime}}\right\|_{L^{2}\left(0, T ; V^{\prime}\right)}^{2}\right)^{\frac{1}{2}}
$$

If $\eta^{i}=\eta^{i 1}+\mathbf{i} \eta^{i 2} \in W(0, T)$ and $\mathbf{n}^{i}=\mathbf{n}^{i 1}+\mathbf{i n}^{i 2} \in W(0, T)$, then definition of inner product is $\left(\eta^{i}, \mathbf{n}^{i}\right)=\left(\eta^{i}, \mathbf{n}^{i}\right)_{V}+\left(\eta^{i \prime}, \mathbf{n}^{i \prime}\right)_{V^{\prime}}$. Respect to the defined norm and inner product, $W(0, T)$ is equipped as a Hilbert space.

Definition 2. A function $\eta^{i}$ is said as weak solution ${ }^{13}$ of nano system in the form of time-depended Schrödinger equation (1) for $i$-th particle $P^{i}, i=1,2, \cdots, N$, if $\eta^{i} \in W(0, T)$ and satisfy

$$
\int_{0}^{T} \int \mathbf{i} \frac{\partial \eta^{i}(t, \mathbf{r})}{\partial t} \sigma^{i} d \mathbf{r} d t=\frac{1}{2} \int_{0}^{T} \int \nabla \eta^{i}(t, \mathbf{r}) \nabla \sigma^{i} d \mathbf{r} d t+\int_{0}^{T} \int[e(t, \mathbf{r})+V(t, \mathbf{r})-g(t)] \eta^{i}(t, \mathbf{r}) \sigma^{i} d \mathbf{r} d t,
$$

where $\forall \sigma^{i} \in \mathscr{D}^{\prime}(0, T)$ by the means of distribution space, $\sigma^{i} \in C^{1}(0, T ; V)$ and $\sigma^{i}(T)=0$.

\section{Solution of nano equation}

Theorem 3. For $\eta^{i}(0, \mathbf{r})=\eta_{0}^{i}(\mathbf{r}) \in V$ of nanoparticle $P^{i}, i=1,2, \cdots, N$, then there exists a unique solution $\eta^{i}(t, \mathbf{r}) \in W(0, T)$ for time-depended Schrödinger nano system (1).

Proof. Faedo-Galerkin method is using to construct an approximate solution for nano system (1). From Gelfand triple spaces, $V \hookrightarrow H$ is compact, then there exists an orthogonal basis of $H$, $\left\{w^{j}\right\}_{j=1}^{\infty}$ consisting of eigenfunctions of $A=-\Delta$, such that $A w^{j}=\lambda{ }^{j} w^{j}$ for all $j, 0<\lambda^{1} \leq \lambda^{2} \leq$ $, \cdots, \lambda^{j} \rightarrow \infty$ as $j \rightarrow \infty$. $G^{i}$ is the orthogonal projection of $H$ (or $V$ ) onto the space spanned by $\left\{w^{1}, \cdots, w^{i^{\prime}}\right\}$. For $1 \leq i \leq N$, an approximate solution is defined for nano equation (1) by

$$
\tilde{\eta}^{i i^{\prime}}(t)=\sum_{j=1}^{i^{\prime}} a^{i j}(t) w^{j}
$$


and $a^{i j}(t)$ is real-valued coefficient function. $i^{\prime}$ is upper index of $i$-th $\tilde{\eta}^{i}$. From $n(t, \mathbf{r})=\sum_{i=1}^{N}\left|\eta^{i}(t, \mathbf{r})\right|^{2}$ to get expansion of $\tilde{e}(t, \mathbf{r})$ and $\tilde{V}(t, \mathbf{r})$ of $e(t, \mathbf{r})$ and $V(t, \mathbf{r})$ using basis functions $\left\{w^{j}\right\}$. Taking $\sigma^{i}=w^{j}$ at weak form (2), then approximate solution $\tilde{\eta}^{i i^{\prime}}(t)$ satisfy ordinary differential equation as

$$
\left\{\begin{array}{l}
\int_{0}^{T} \int \mathbf{i} \frac{\partial \tilde{\eta}^{i i^{\prime}}}{\partial t} w^{j} d \mathbf{r} d t=\frac{1}{2} \int_{0}^{T} \int \nabla \tilde{\eta}^{i i^{\prime}} \nabla w^{j} d \mathbf{r} d t+\int_{0}^{T} \int[\tilde{e}(t, \mathbf{r})+\tilde{V}(t, \mathbf{r})-g(t)] \tilde{\eta}^{i i^{\prime}} w^{j} d \mathbf{r} d t \\
\tilde{\eta}^{i i^{\prime}}(0)=\tilde{\eta}_{0}^{i}, \quad 1 \leq i \leq N
\end{array}\right.
$$

Substitute $\tilde{\eta}^{i i^{\prime}}$ as approximate form in (3), from standard theory of ODE, ensure a unique local solution $\left\{\tilde{\eta}^{i i^{\prime}}\right\}, i=1,2, \cdots, N$ for approximate system (3). For $\tilde{\eta}_{0}^{i i^{\prime}}$, there exists a $\bar{\eta}_{0}^{i} \in V$ such that

$$
\tilde{\eta}_{0}^{i i^{\prime}} \rightarrow \bar{\eta}_{0}^{i} \text { strongly in } \quad H_{0}^{1}(\Omega), \quad \text { as } i^{\prime} \rightarrow \infty
$$

Take analogous argument for $\left\{\tilde{\eta}^{i i^{\prime}}\right\}$, there exist a function $\bar{\eta}^{i} \in V$, that $\left\|\tilde{\eta}^{i i^{\prime}}-\bar{\eta}^{i}\right\|_{V} \rightarrow 0$ as $i^{\prime} \rightarrow \infty$. That is, approximate solution $\tilde{\eta}^{i i^{\prime}}$ is bounded in $L^{\infty}(0, T ; V)$. Suppose that $\left\{\tilde{\eta}^{i i^{\prime}}\right\}$ and $\left\{\tilde{\eta}^{j i^{\prime}}\right\}$ are two solutions of nano equation (1) for initials $\eta_{0}^{i}$ and $\eta_{0}^{j}$. $\tilde{e}^{i i^{\prime}}=\tilde{e}\left(t, \mathbf{r}, \eta^{i i^{\prime}}\right), \tilde{V}^{i i^{\prime}}=\tilde{V}\left(t, \mathbf{r}, \eta^{i i^{\prime}}\right)$ for solution $\eta^{i i^{\prime}}$, and $\tilde{e}^{j i^{\prime}}=\tilde{e}\left(t, \mathbf{r}, \eta^{j i^{\prime}}\right), \tilde{V}^{j i^{\prime}}=\tilde{V}\left(t, \mathbf{r}, \eta^{j i^{\prime}}\right)$ for solution $\eta^{j i^{\prime}}$. To estimate the external force as

$$
\begin{aligned}
& \tilde{e}^{i i^{\prime}} \eta^{i i^{\prime}}-\tilde{e}^{j i^{\prime}} \eta^{j i^{\prime}} \\
= & \int \frac{\sum_{i=1}^{N}\left|\eta^{i i^{\prime}}\right|^{2}}{\left|\mathbf{r}-\mathbf{r}^{\prime}\right|} d \mathbf{r}^{\prime} \eta^{i i^{\prime}}-\int \frac{\sum_{j=1}^{N}\left|\eta^{j i^{\prime}}\right|^{2}}{\left|\mathbf{r}-\mathbf{r}^{\prime}\right|} d \mathbf{r}^{\prime} \eta^{j i^{\prime}} \\
= & \tilde{e}^{i i^{\prime}}\left(\eta^{i i^{\prime}}-\eta^{j i^{\prime}}\right)+\left(\tilde{e}^{i i^{\prime}}-\tilde{e}^{j i^{\prime}}\right) \eta^{j i^{\prime}} \\
= & \tilde{e}^{i i^{\prime}}\left(\eta^{i i^{\prime}}-\eta^{j i^{\prime}}\right)+\int \frac{\sum_{i=1}^{N}\left|\eta^{i i^{\prime}}\right|^{2}-\sum_{j=1}^{N}\left|\eta^{j i^{\prime}}\right|^{2}}{\sum_{i, j}^{N}\left|\eta^{i i^{\prime}}-\eta^{\prime}\right|} d \mathbf{r}^{\prime} \eta^{j i^{\prime}}|| \eta^{i i^{\prime}}+\eta^{j i^{\prime} \mid} \\
= & \tilde{e}^{i i^{\prime}}\left(\eta^{i i^{\prime}}-\eta^{j i^{\prime}}\right)+\int \frac{\mathbf{r}^{\prime}-\mathbf{r}^{\prime} \mid}{\mathbf{r}^{\prime}} \eta^{j i^{\prime}} .
\end{aligned}
$$


Then to have formula

$$
\left\|\tilde{e}^{i i^{\prime}} \eta^{i i^{\prime}}-\tilde{e}^{j i^{\prime}} \eta^{j i^{\prime}}\right\|^{2} \leq\left\|\tilde{e}^{i i^{\prime}}\right\|_{H}^{2}+\left\|\eta^{i i^{\prime}}-\eta^{j i^{\prime}}\right\|_{H}^{2}+\left\|\tilde{e}^{i i^{\prime}}-\tilde{e}^{j i^{\prime}}\right\|_{H}^{2}+\left\|\eta^{j i^{\prime}}\right\|_{H}^{2} .
$$

Further to estimate

$$
\left\|\tilde{V}^{i i^{\prime}} \eta^{i i^{\prime}}-\tilde{V}^{j i^{\prime}} \eta^{j i^{\prime}}\right\|^{2} \leq\left\|\tilde{V}^{i i^{\prime}}\right\|_{H}^{2}+\left\|\eta^{i i^{\prime}}-\eta^{j i^{\prime}}\right\|_{H}^{2}+\left\|\tilde{V}^{i i^{\prime}}-\tilde{V}^{j i^{\prime}}\right\|_{H}^{2}+\left\|\eta^{j i^{\prime}}\right\|_{H}^{2} .
$$

Hence, by sum of $w^{j}$ on coefficients $a^{i j}(t)$ from $j=1$ to $i^{\prime}$ (same as replacing $w^{j}$ to $\tilde{\eta}^{i i^{\prime}}$ ) at weak form (2) to get

$$
\begin{aligned}
& \frac{\mathbf{i}}{2} \frac{d}{d t}\left\|\tilde{\eta}^{i i^{\prime}}-\tilde{\eta}^{j i^{\prime}}\right\|_{H}^{2}+g(t)\left\|\tilde{\eta}^{i i^{\prime}}-\tilde{\eta}^{j i^{\prime}}\right\|_{V}^{2} \\
\leq & \frac{1}{2}\left\|\tilde{\eta}^{i i^{\prime}}-\tilde{\eta}^{j i^{\prime}}\right\|_{V}^{2}+2\left\|\eta^{i i^{\prime}}-\eta^{j i^{\prime}}\right\|_{H}^{2}+\left(\left\|\tilde{e}^{i i^{\prime}}-\tilde{e}^{j i^{\prime}}\right\|_{H}^{2}+\left\|\tilde{V}^{i i^{\prime}}-\tilde{V}^{j i^{\prime}}\right\|_{H}^{2}\right) \\
& +\left(\left\|\tilde{V}^{i i^{\prime}}\right\|_{H}^{2}+\left\|\tilde{e}^{i i^{\prime}}\right\|_{H}^{2}+2\left\|\eta^{j i^{\prime}}\right\|_{H}^{2}\right) .
\end{aligned}
$$

Set $I^{i j}(t)=\left\|\tilde{\eta}^{i i^{\prime}}-\tilde{\eta}^{j i^{\prime}}\right\|_{H}^{2}+\left\|\tilde{\eta}^{i i^{\prime}}-\tilde{\eta}^{j i^{\prime}}\right\|_{V}^{2}$, and $C(t)=\left\|\tilde{e}^{i i^{\prime}}\right\|_{H}^{2}+\left\|\tilde{V}^{i i^{\prime}}\right\|_{H}^{2}+2\left\|\eta^{j i^{\prime}}\right\|_{H}^{2}$, by the positive of second term in left side, and polynomial convergence of expansions $\tilde{e}(t, \mathbf{r})$ and $\tilde{V}(t, \mathbf{r})$ in third term of right side at above formula to sure

$$
\mathbf{i} \frac{d}{d t} I^{i j}(t) \leq I^{i j}(t)+C(t)
$$

Set $I^{i j}(0)=\left\|\tilde{\eta}_{0}^{i i^{\prime}}-\tilde{\eta}_{0}^{j i^{\prime}}\right\|_{H}^{2}+\left\|\tilde{\eta}_{0}^{i i^{\prime}}-\tilde{\eta}_{0}^{j i^{\prime}}\right\|_{V}^{2}$, Gronwall inequality yield that

$$
I^{i j}(t) \leq e^{t} I^{i j}(0)+\int_{0}^{t} C(t) e^{t-t^{\prime}} d t^{\prime}, \quad 0 \leq t^{\prime} \leq t
$$

It implied $\tilde{\eta}^{i i^{\prime}} \rightarrow \tilde{\eta}^{j i^{\prime}}$ in $L^{2}(0, T ; H)$ and $L^{2}(0, T ; V)$ as $i^{\prime} \rightarrow \infty$. Taking $\tilde{\eta}^{j i^{\prime}}=\eta^{i}$ to find that $\tilde{\eta}^{i i^{\prime}} \rightarrow \eta^{i}$ in $C(0, T ; H)$. Thus, inclusion $C(0, T ; H) \subset W(0, T)$ to ensure Theorem 3 . 


\section{Applying quantum control to nano system}

Consider theoretical existence of quantum optimal control for nano particle at catalysis, crystal surface. For control $g(t)$, set corresponding optimal control $g^{*}(t)$. Suppose $\mathscr{G}=L^{2}(0, T)$ is the space of control $g(t)$, and $\mathscr{G}_{a d}$ is a admissible set of $\mathscr{G}$. Set ground state of $i$-th nano particle $P^{i}$ as $\eta^{i}(0)=\eta_{0}$. Consider cost function of $i$-th nano particle $P^{i}$ for time-depended Schrödinger system (1) is in the form of

$$
J^{i}(g)=\varepsilon^{1}\left\|\eta_{f}^{i}(g)-\eta_{d}^{i}\right\|_{H}^{2}+\varepsilon^{2}\|g\|_{\mathscr{G}}^{2}
$$

for $\forall g \in \mathscr{G}_{a d}$. In criteria function (4), $\eta_{d}^{i}$ is target state, $\left.\eta_{f}^{i}(g)\right)$ is observed final state of nano particle $P^{i}$ at final time $t_{f} . \varepsilon^{i}, i=1,2$ are weighted coefficients for balancing the values of inhere cost and running cost. Total cost function $J(g)=\sum_{i=1}^{N} J^{i}(g)$ for all nano particles at a certain matter surface.

Two fundamental problems of quantum optimal control for nano quantum system:

i). find quantum optimal control $g^{*}$ for nano system (1).

ii). find Eular-Lagrange system for $g^{*}$.

For $i$-th nanoparticle $P^{i}, g^{*}(t)$ is said as quantum optimal control for nano system (1) to cost function (4).

Theorem 4. For $\eta_{0}^{i} \in V$ of $i$-th nano particle $P^{i}, n=1,2, \cdots, N$, if $\mathscr{G}_{a d}$ is closed convex (bounded) admissible subset of $\mathscr{G}$, then there exists at least one quantum optimal control $g^{*}$ of nano system (1) subject to cost function (4).

Proof. Set $J=\inf _{g \in \mathscr{G}_{a d}} J^{i}(g)$, since $\mathscr{G}_{a d}$ is non-empty, there is a sequence $\left\{g^{k}\right\}$ in $\mathscr{G}_{a d}$ such that $\inf _{g \in \mathscr{G}_{a d}} J^{i}(g)=\lim _{k \rightarrow \infty} J\left(g^{k}\right)=J$. Since $\left\{J^{i}(g)\right\}$ is bounded in $\mathbf{R}^{+}$, and $\mathscr{G}_{a d}$ is closed and convex (bounded) subset of $\mathscr{G}$, there exist a subsequences $\left\{g^{k^{\prime}}\right\}$ of $\left\{g^{k}\right\}$ can be extracted, and exist a $g^{*} \in \mathscr{G}_{a d}$, such that

$$
g^{k^{\prime}} \rightarrow g^{*} \text { weakly in } \mathscr{G} \quad \text { as } k^{\prime} \rightarrow \infty \text {. }
$$


From existence theorem 1 of weak solution to get estimate $\left\|\eta^{i}\right\|_{H}^{2}+\left\|\eta^{i}\right\|_{V}^{2}$ is bounded for $\eta^{i}$ of $i$-th nano particle $P^{i}$. For control $g^{k^{\prime}}$, from boundedness of $\mathscr{G}_{a d}$ that

$$
\eta^{i}\left(g^{k^{\prime}}\right) \text { is bounded in } L^{2}(0, T ; H) \cap L^{2}(0, T ; V)
$$

Setting $\eta^{i *}=\eta^{i}\left(g^{*}\right)$, there exist a subsequence $\left\{\eta^{i}\left(g^{k^{\prime \prime}}\right)\right\}$ of $\left\{\eta^{i}\left(g^{k^{\prime}}\right)\right\}$, and a $\eta^{i *} \in W(0, T)$ such that

$$
\eta^{i}\left(g^{k^{\prime \prime}}\right) \rightarrow \eta^{i *} \text { weakly in } L^{2}(0, T ; H) \cap L^{2}(0, T ; V)
$$

as $k^{\prime \prime} \rightarrow \infty$. Since the embedding $V \hookrightarrow H$ is compact, from Aubin-Lions-Temam theorem, then there is $\bar{\eta}^{i} \in H$ that

$$
\eta^{i}\left(g^{k^{\prime \prime}}\right) \rightarrow \bar{\eta}^{i} \quad \text { strongly in } L^{2}(0, T ; H), \quad \text { as } k^{\prime \prime} \rightarrow \infty
$$

and get the convergences for $\eta^{i}$ as

$$
\left\{\begin{array}{c}
\frac{\partial \eta^{i}\left(g^{k^{\prime \prime}}\right)}{\partial t} \rightarrow \frac{\partial \bar{\eta}^{i}}{\partial t} \quad \text { weakly in } L^{2}\left(0, T ; V^{\prime}\right) \\
\nabla \eta^{i}\left(g^{k^{\prime \prime}}\right) \rightarrow \nabla \bar{\eta}^{i} \quad \text { weakly in } L^{2}(0, T ; H)
\end{array}\right.
$$

as $k^{\prime \prime} \rightarrow \infty$. Set $\eta^{i k^{\prime \prime}}=\eta^{i}\left(g^{k^{\prime \prime}}\right), \eta_{0}^{i k^{\prime \prime}}=\eta_{0}^{i}$, therefore, for $i$-th nano particle $P^{i}$, by taking test function $w^{j}=\sigma^{i}$ in (3), by the definition of weak form (2) to find

$$
\left\{\begin{array}{l}
\int_{0}^{T} \int \mathbf{i} \frac{\partial \eta^{i k^{\prime \prime}}}{\partial t} \sigma^{i} d \mathbf{r} d t=\frac{1}{2} \int_{0}^{T} \int \nabla \eta^{i k^{\prime \prime}} \nabla \sigma^{i} d \mathbf{r} d t+\int_{0}^{T} \int[e(t, \mathbf{r})+V(t, \mathbf{r})-g(t)] \eta^{i k^{\prime \prime}} \sigma^{i} d \mathbf{r} d t \\
\eta^{i k^{\prime \prime}}(0)=\eta_{0}^{i k^{\prime \prime}}, \quad 1 \leq i \leq N
\end{array}\right.
$$

If using (5), (6), and taking $k^{\prime \prime} \rightarrow \infty$ to yield that

$$
\left\{\begin{array}{l}
\int_{0}^{T} \int \mathbf{i} \frac{\partial \bar{\eta}^{i}}{\partial t} \sigma^{i} d \mathbf{r} d t=\frac{1}{2} \int_{0}^{T} \int \nabla \bar{\eta}^{i} \nabla \sigma^{i} d \mathbf{r} d t+\int_{0}^{T} \int[e(t, \mathbf{r})+V(t, \mathbf{r})-g(t)] \bar{\eta}^{i} \sigma^{i} d \mathbf{r} d t \\
\bar{\eta}^{i}(0)=\eta_{0}^{i}, \quad 1 \leq i \leq N,
\end{array}\right.
$$


For $\forall \sigma^{i} \in C^{1}(0, T ; V)$. It inferred that $\bar{\eta}^{i}$ is a solution of (1) in the sense of distribution $\mathscr{D}^{\prime}(0, T)$ on $(0, T)$. From uniqueness of weak solution for nano system to attain $\bar{\eta}^{i}=\eta^{i}\left(g^{*}\right)$. For $\eta^{i}$ to get two convergences as

$$
\begin{aligned}
& \eta^{i}\left(g^{k^{\prime \prime}}\right) \rightarrow \eta^{i}\left(g^{*}\right) \quad \text { strongly in } \quad L^{2}(0, T ; H), \\
& \eta_{f}^{i}\left(g^{k^{\prime \prime}}\right) \rightarrow \eta_{f}^{i}\left(g^{*}\right) \quad \text { strongly in } \quad H, \quad \text { as } k^{\prime \prime} \rightarrow \infty .
\end{aligned}
$$

Since the norm $\|\cdot\|_{L^{2}(\Omega)}$ are lower semi-continuous in weak topology of $L^{2}(\Omega)$, for $i$-th nano particle $P^{i}$

$$
\liminf _{k^{\prime \prime} \rightarrow \infty}\left\|\eta_{f}^{i}\left(g^{k^{\prime \prime}}\right)-\eta_{d}^{i}\right\|_{H}^{2} \geq\left\|\eta_{f}^{i}\left(g^{*}\right)-\eta_{d}^{i}\right\|_{H}^{2}
$$

Vice versa, from weak convergence (5) that $\liminf _{k^{\prime \prime} \rightarrow \infty}\left(g^{k^{\prime \prime}}, g^{k^{\prime \prime}}\right) \mathscr{G} \geq\left(g^{*}, g^{*}\right) \mathscr{G}$. For cost function (4), $J=\liminf _{k^{\prime \prime} \rightarrow \infty} J^{i}\left(g^{k^{\prime \prime}}\right) \geq J^{i}\left(g^{*}\right)$, and $J^{i}\left(g^{*}\right)=\inf _{g \in \mathscr{G}_{a d}} J^{i}(g)$. That is, $g^{*}$ is quantum optimal control of nano system (1) subject to criteria function (4). It is Theorem 4.

For quadratic cost function (4), the existence of Gâteaux differentiable of $J^{i}(g)$ on control variable $g$ make us to rewrite the first order necessary optimality condition $D J^{i}\left(g^{*}\right)\left(g-g^{*}\right) \geq 0$ in term of solution for adjoint system. One can derive the optimality system of quantum optimal control $g^{*}$ for nano system (1) to cost function (4).

Theorem 5. For $\eta_{0}^{i}(\mathbf{r}) \in V$ of control problem for nano system (1) to cost function (4), if $\mathscr{G}_{a d}$ is closed convex (bounded) admissible subset of $\mathscr{G}$, then quantum optimal control $g^{*}$ is characterized by Eular-Lagrange system as

$$
\begin{aligned}
& \left\{\begin{array}{l}
\mathbf{i} \frac{\partial}{\partial t} \eta^{i}(t, \mathbf{r})=-\frac{1}{2} \Delta \eta^{i}(t, \mathbf{r})+\left[e(t, \mathbf{r})+V(t, \mathbf{r})-g^{*}(t)\right] \eta^{i}(t, \mathbf{r}) \quad \text { in } \mathrm{Q}, \\
\eta^{i}\left(0, g^{*}\right)=\eta_{0}^{i} \quad \text { on } \Omega .
\end{array}\right. \\
& \left\{\begin{array}{c}
\mathbf{i} \frac{\partial}{\partial t} \mathbf{n}^{i}(t, \mathbf{r})=-\frac{1}{2} \Delta \mathbf{n}^{i}(t, \mathbf{r})+[e(t, \mathbf{r})+V(t, \mathbf{r})] \mathbf{n}^{i}(t, \mathbf{r}) \quad \text { in } \mathrm{Q}, \\
\mathbf{n}^{i}\left(T, g^{*}\right)=\eta_{f}^{i}\left(g^{*}\right)-\eta_{d}^{i} \quad \text { on } \Omega .
\end{array}\right. \\
& \int_{0}^{T} \sigma^{*} \mathbf{n}^{i}\left(g^{*}\right)\left(g-g^{*}\right) d \mathbf{r} d t+\left(g^{*}, g-g^{*}\right) \mathscr{G} \geq 0 \quad \forall g \in \mathscr{G}_{a d} .
\end{aligned}
$$


In here, $\mathbf{n}^{i} \in W(0, T)$ is solution of adjoint system (8) corresponding to $\eta^{i}$ of nano system (7). (9) is necessary optimality condition for quantum optimal control $g^{*}$ of $i$-th nano particle $P^{i}, i=$ $1,2, \cdots, N . \sigma^{*}$ is conjugate operator of $\sigma$ in $\sigma\left(\eta^{i}\right)=g^{*} \eta^{i}$.

\section{Discussion}

Practically, theoretic found optimal control $g^{*}$ can be executed in laboratory by the means of control adjustment or confinements. This kind of physical control had been taken as: control particle-type (atomic number, Fermi energy, potential energy, electron density); control particle size (number of atoms, ground state molecules, lattice constants); quantum number selection rule for principle and angular quantum number. For instance, metal nano particle $1-100 \mathrm{~nm}$ contain $10^{2}-10^{8}$ atoms, nobel metal nano particle silver $A g$, gold $A u$ take lattice constants 0.408, 0.407, principle quantum number $n=5, n=6$, Fermi level $\mu=5.49 \mathrm{eV}, \mu=5.53 \mathrm{eV}$, et al. Our work is based upon those experimental results, consider the theoretic control theory as purpose to evident existing physical control conclusion and provide theoretical support for real-time computer aided lab control.

\section{Conclusions}

In summary, at this work, quantum control for nano-particles at matter (catalysis, crystal, metal) surface is proposed initially and theoretically using the density function theory of time-depended Schrödinger equation. Through applying the DFT approach to TDSE, optimal control theory is worked as consideration to manipulate the nano-particles appeared at a certain surface (no chemical reaction take place). Compare to the study of particles at surface governed by Schrödinger equation, density function theory is a new tool for controlling at nanoscale under the optical equipment by accessing the successful control conclusion on molecule and atom scales. Theoretical control, computational control and experimental control of nano-particles at surface would be extremely interesting work in the future. 


\section{Acknowledgement}

The author would like to express sincere thanks to 2014 ACS National Meeting for poster. ${ }^{14}$

\section{References}

(1) Wang, Q. F. ACS National Meeting \& Exposition 2009, Washington D.C., Poster

(2) Horikoshi, S.; Serpone, N. Introduction to Nano Particles, Microwaves in Nano-particles, 2013, Wiley-VCH Verlag GmbH \& Co KGaA.

(3) Morgan III, J. D. Atomic, Molecular, \& Optical Physics Handbooks, 1996233

(4) Thomas, L. H. Proc. Camb. Phil. Soc. 1927, 23, 542

(5) Fermi, E. Rend. Accad. Naz. Lincei 1927 6, 602

(6) Dirac, P. A. M. Proc. Camb. Phil. Soc. 1930 26, 376

(7) Weizsäcker, C. F. Zeitschrift für Physik 193596 (7-8), 431

(8) Hohenberg, P.; Kohn, W. Physical Review 1964 136, B864

(9) Kohn, W.; Sham, I. J. Physical Review 1965 140, A1133

(10) Harrison, N. M. An Introduction to Density Function Theory 2002

(11) Mack, H. G. Combined quantum mechanics and classical electrodynamics mulit-scale approach for the calculation of SERS spectra (A brief survey), 2013.

(12) Adams, R. Sobolev Spaces 1975, New York, Academic Press.

(13) Lions, J. L. Optimal Control of Systems Governed by Partial Differential Equations 1971, Berlin-Heidelberg-New York, Springer-Verlag.

(14) Wang, Q. F. ACS National Meeting \& Exposition 2014, San Francisco, Poster 